\title{
Assessment to the Antifungal Effects in vitro and the Ocular Pharmacokinetics of Solid-Lipid Nanoparticle in Rabbits
}

\author{
Zhen Liang \\ Zhen Zhang \\ Jingjing Yang \\ Ping Lu \\ Tianyang Zhou \\ Jingguo $\mathrm{Li}$ (D) \\ Junjie Zhang
}

Henan Eye Hospital, Henan Provincial People's Hospital \& People's Hospital of Zhengzhou University, Zhengzhou,

People's Republic of China
Correspondence: Jingguo Li; Junjie Zhang Henan Eye Hospital, Henan Provincial People's Hospital \& People's Hospital of Zhengzhou University, Number 7 Weiwu Road, Zhengzhou, People's Republic of China

Email lijingguo@zzu.edu.cn; zhangjunjie@zzu.edu.cn
Introduction: Fungal keratitis (FK) remains a severe sight-threatening disease, and case management is difficult due to ocular intrinsic barriers and drug shortages. Econazole (ECZ), a broad-spectrum antifungal agent, is limited in ocular applications due to the poor water solubility and strong irritant property.

Methods: We successfully prepared solid-lipid nanoparticle-based ECZ eye drops (E-SLNs) by microemulsion method, and the physicochemical properties of E-SLNs were investigated. Corneal permeability, antifungal ability against Fusarium spp., irritation and bioavailability compared to ECZ Suspension (E-Susp) were evaluated in vitro and in vivo.

Results: E-SLNs were a uniform and stable system which had an average particle size of 19 $\mathrm{nm}$ and a spherical morphology. E-SLNs also exhibited controlled release, enhanced antifungal activity without irritation. The pharmacokinetic analysis in vivo confirmed that E-SLNs showed an improved ocular bioavailability and the drug concentration in the cornea were above minimum inhibitory concentration (MIC) for $3 \mathrm{~h}$ after single administration.

Conclusion: The E-SLNs colloid system is a promising therapeutic approach for fungal keratitis and could serve as a candidate strategy for other ocular diseases.

Keywords: fungal keratitis, solid-lipid nanoparticles, econazole, topical administration, corneal penetration

\section{Introduction}

Fungal keratitis (FK) is a severe sight-threatening disease found worldwide with a high probability of blindness and eye loss. ${ }^{1,2}$ In recent years, FK morbidity has shown an increasing trend in developing countries in tropical and subtropical climates, mainly related to agricultural corneal trauma, the wearing of contact lenses, and the use of broad-spectrum antibiotics and immunosuppressive agents. ${ }^{3}$ The main pathogenic fungi that cause this condition are Fusarium spp. and Aspergillus spp. in China. ${ }^{4}$ Fungi can penetrate the cornea and invade the aqueous humor, making them very difficult to be eradicated. ${ }^{5}$

The topical administration of antifungal drugs is usually the top choice of treatment of fungal keratitis. ${ }^{2}$ Natamycin is the first-line treatment and the only medication approved by the US Food and Drug Administration to treat FK. However, due to poor corneal penetration, case management is difficult, and a long course of therapy is needed. ${ }^{6}$ Natamycin is instilled every hourly or two hourly for 4-6 weeks in clinical treatment. ${ }^{7}$ Despite this, there are still $40 \%$ of patients with FK have to receive other treatment. ${ }^{1}$ So, it is urging to seek out 
alternative drugs. Some ophthalmic formulations containing ECZ could effectively inhibit Fusarium spp. isolated from patients with $\mathrm{FK}^{8}$ and show better antifungal activity compared to natamycin. ${ }^{9}$ However, no ophthalmic formulations of ECZ are used in clinical practice due to its poor water solubility ${ }^{10}$ (approximately $3 \mu \mathrm{g}$ / $\mathrm{mL}$ at $25^{\circ} \mathrm{C}$ ) and irritant property. Therefore, it is desirable to formulate ECZ into a suitable system that can deliver it to achieve an efficient concentration in the cornea after topical administration.

Due to the presence of anatomical and physiological barriers, ocular drug delivery is challenging. Topical administration in the form of eye drops is preferred for treating anterior segment diseases, such as infections, inflammation, and glaucoma, as it is convenient and allows the local delivery of drugs. However, poor ocular bioavailability $(<5 \%)$ is observed after topical drug administration due to tear turnover, nasolacrimal drainage, reflex blinking and epithelial tight junctions, namely, the tear-film barrier and corneal barrier. ${ }^{11}$ Thus, several applications per day are required to achieve therapeutic effect. However, frequent topical application can cause irritation, side effects and poor patient compliance. ${ }^{12}$

Solid lipid nanoparticles (SLNs) were developed as a particulate delivery system alternative to the few other colloidal carriers. SLNs can provide encapsulation and protection of labile drugs from degradation, improve bioavailability of poorly water-soluble drugs, modulate the drug release and drug targeting, and are convenient for large-scale production and sterilization. ${ }^{13,14}$ SLNs have received special attention from ophthalmic researchers with advantages over conventional dosage formulations in enhancing corneal drug absorption, increasing ocular bioavailability, prolonging the ocular retention time, and providing controlled drug release. ${ }^{15,16}$

Accordingly, the aim of this study was to design a SLNs loaded with ECZ (E-SLNs) to improve the bioavailability of ECZ. Hence, E-SLNs were prepared and characterized in term of particle size, zeta potential, morphology, drug entrapment efficiency (EE), and in vitro drug release. The in vitro antifungal activity of E-SLNs against Fusarium spp. was tested. Ex vivo permeation, in vivo ocular irritation and ocular pharmacokinetic profiles were also investigated.

\section{Materials and Methods}

\section{Animals and Materials}

Healthy New Zealand white rabbits free of eye diseases weighing $2.0 \sim 2.5 \mathrm{~kg}$ (3 months of age) were purchased from HuaXing Laboratory Animal Company, Huiji District, Zhengzhou, Henan, China. All animal care and experimental protocols were approved by the Ethical Committee of Experimental Animal Care of Henan Eye Institute (HENNCA-2017-22) and complied with National Institutes of Health guidelines. All procedures in the study conformed to the ARVO statement.

Econazole (ECZ) nitrate was purchased from Dalian Meilun Biotechnology Co., Ltd. (Dalian, China). The ECZ base was prepared from ECZ nitrate as previously described. ${ }^{10}$ Tween 80 was purchased from Sichuan Jinshan Pharmaceutical Co., Ltd. (Sichuan, China), and tripalmitin was purchased from TCI Chemical Industry Development Co., Ltd. (Shanghai, China). Dialysis bags (MW cutoff: $3500 \mathrm{kDa}$ ) were purchased from Shanghai Green Bird Technology Development Co., Ltd. (Shanghai, China) and stored in $1 \mathrm{mM}$ ethylenediaminetetraacetic acid (EDTA) aqueous solution prior to use. Amicon ${ }^{\circledR}$ Ultra-4 10K centrifugal filter devices were purchased from Millipore Investment Co., Ltd. (USA). Methanol [high-performance liquid chromatography (HPLC) grade] was purchased from Merck Investment Co., Ltd. (Germany), and ethyl acetate (HPLC grade) was purchased from Fisher Scientific Company (USA). Water for injection was used for the ophthalmic preparations. All other reagents were of analytical grade.

\section{Preparation and Characterization of the E-SLNs} Preparation of E-SLNs and ECZ Suspension (E-Susp) The E-SLNs were prepared by the microemulsion method. ${ }^{17}$ The oil phase, including ECZ (0.06 g), tripalmitin $(0.06 \mathrm{~g})$ and Tween $80(0.6 \mathrm{~g})$, was heated to $70^{\circ} \mathrm{C}$ by a magnetic stirrer $(1000 \mathrm{rpm})$. Then, deionized water containing glycerol $(1.6 \mathrm{~g})$ at the same temperature was injected into the oil phase by means of a syringe under magnetic stirring to obtain the oil/water microemulsion solution. Subsequently, the hot $\mathrm{o} / \mathrm{w}$ microemulsion was cooled to room temperature while maintaining the mechanical stirring until SLNs were formed.

The ECZ suspension (E-Susp) was prepared as previously described. ${ }^{10}$

\section{Particle Size, Polydispersity Index (PDI) and Zeta Potential}

The average particle size, PDI and zeta potential of the prepared E-SLNs formulations were determined using a Malvern Zetasizer 4 (Nano ZS, Zen 3600; Malvern 
instruments, Malvern, UK) after suitable dilution. Each value in the results was the average of three measurements.

The average particle size of E-Susp was determined as noted above.

\section{Entrapment Efficiency (EE)}

The concentration of ECZ (free drug) was measured as a criterion for the EE of the prepared E-SLNs. An ultracentrifugal filter device was used to separate the free drug as follows: $4 \mathrm{~mL}$ of undiluted E-SLNs was added to the filter device, and the device was centrifuged at $4000 \mathrm{rpm}$ for $20 \mathrm{~min}$. Then, the free drug in the centrifuge tube was determined by an HPLC system. The column was a reversed-phase $\mathrm{C} 18$ column $(3.0 \times 150 \mathrm{~mm}, 3.5 \mu \mathrm{m})$. The mobile phase consisted of methanol and deionized water $(80: 20 \mathrm{v} / \mathrm{v})$ at a detection wavelength of $225 \mathrm{~nm}$. The flow rate was $0.5 \mathrm{~mL} / \mathrm{min}$, and the column temperature was maintained at $40^{\circ} \mathrm{C}$. The EE was calculated as follows: ${ }^{18}$

$$
\mathrm{EE}=\left(1-\left(\mathrm{C}_{\mathrm{F}} \times \mathrm{V}_{\mathrm{F}}\right) /\left(\mathrm{C}_{0} \times \mathrm{V}_{0}\right)\right) \times 100 \%
$$

where $\mathrm{C}_{0}$ and $\mathrm{V}_{0}$ are the concentration of drug in the original solution and the volume of the original solution, respectively. $C_{F}$ and $V_{F}$ are the concentration of drug in the filtrate and its volume in the centrifuge tube after ultrafiltration of the original solution, respectively.

\section{The Ratio of E-SLNs in the Colloid Systems}

As the surfactant was added to the formulation when preparing the E-SLNs by the microemulsion method, the colloid system could contain micelles that encapsulated ECZ (E-micelles) and free drug in addition to E-SLNs. Equation 2 expressed that the amount of ECZ in the colloid system was equal to the sum of the free drug in the original solution, the ECZ in the SLNs and the ECZ in the micelles. To determine the ratio of ECZ in E-SLNs, the colloid system should be disrupted by dilution to a concentration lower than the critical micelle concentration (CMC). Equation 3 expressed that the concentration of ECZ in the dilution was equal to the sum of the free drug in the dilution and ECZ in the SLNs as the micelles were completely disintegrated after the solution was diluted.

Ultracentrifugal filter devices were used to separate the free drug as follows: the original E-SLNs dispersion and the diluted E-SLNs dispersion were added to the filter device, and the device was centrifuged at $4000 \mathrm{rpm}$ for $20 \mathrm{~min}$. Next, the free drug was filtered in a centrifuge tube. ECZ concentrations in the original, diluted E-SLNs dispersion and filtrate were determined by HPLC as described above. The ratio of ECZ in E-SLNs in the colloid systems was calculated as follows:

$$
\begin{gathered}
\mathrm{C}_{0} \times \mathrm{V}_{0}=\mathrm{C}_{\mathrm{F} 1} \times \mathrm{V}_{\mathrm{F} 1}+\mathrm{M}_{\mathrm{E}-\mathrm{SLNs}}+\mathrm{M}_{\mathrm{E}-\text { Micelle }} \\
(1 / 1600) \times \mathrm{C}_{0} \times \mathrm{V}_{0}=\mathrm{C}_{\mathrm{F} 2} \times \mathrm{V}_{\mathrm{F} 2}+(1 / 1600) \times \mathrm{M}_{\mathrm{E}-\mathrm{SLNs}} \\
\mathrm{R}_{\mathrm{E}-\mathrm{SLNs}}=\left(\mathrm{M}_{\mathrm{E}-\mathrm{SLNs}} /\left(\mathrm{C}_{0} \times \mathrm{V}_{0}\right)\right) \times 100 \%
\end{gathered}
$$

where $\mathrm{C}_{0}$ is the concentration of ECZ in the original solution and $\mathrm{V}_{0}$ is the volume of the original solution (4 mL). Additionally, $\mathrm{C}_{\mathrm{F} 1}$ and $\mathrm{V}_{\mathrm{F} 1}$ are the concentration of drug in the filtrate and its volume in the centrifuge tube after ultrafiltration of the original solution, respectively. $\mathrm{C}_{\mathrm{F} 2}$ and $\mathrm{V}_{\mathrm{F} 2}$ are the concentration of drug in the filtrate and its volume in the centrifuge tube after ultrafiltration of the diluted solution, respectively. $\mathrm{M}_{\mathrm{E}-\mathrm{SLNs}}$ and $\mathrm{M}_{\mathrm{E}-\text { Micelle }}$ are the amount of ECZ loaded in the SLNs and micelles in the $4 \mathrm{~mL}$ of original solution, respectively. Finally, $\mathrm{R}_{\mathrm{E}-\mathrm{SLNs}}$ is the percent ratio of the ECZ amount loaded in SLNs and in the original solution.

\section{Transmission Electron Microscopy (TEM)}

A TEM investigation was performed to investigate the morphological characteristics of the E-SLNs. A drop of E-SLNs was mounted on a carbon-coated grid and left for 2 min to allow better adsorption onto the carbon film, and filter paper was used to remove excess liquid. A drop of phosphotungstic acid (2\%) was added, and then the sample was examined by TEM (Quanta 400F, FEI, USA).

\section{Differential Scanning Calorimetry (DSC)}

Thermograms of the different samples were obtained using a DSC Q200 instrument (TA Instruments, New Castle, USA). The instrument was calibrated with indium (calibration standard, purity 99.999\%) for the melting point and heat of fusion. Samples $(2-3 \mathrm{mg})$ were heated in crimped aluminum pans from $20-80^{\circ} \mathrm{C}$ at a heating rate of $10^{\circ} \mathrm{C} / \mathrm{min}$. Analyses were carried out under an inert nitrogen purge $(20 \mathrm{~mL} / \mathrm{min})$, and an empty alumina pan was used as a reference in every case. The thermal analyses were carried out on ECZ, on freeze-dried E-SLNs, and on a physical mixture of ECZ, tripalmitin. The experiments were performed in triplicate.

\section{In vitro Drug Release Studies}

The release rate of ECZ from the E-SLNs dispersion was determined using a dialysis bag diffusion technique, and 
artificial tear solution containing $0.05 \%$ Tween 80 was selected as the release medium. One milliliter of E-SLNs was pipetted into a dialysis bag (MW cutoff: $3500 \mathrm{Da}$ ), which was then sealed at both ends. Then the dialysis bag was placed in $150 \mathrm{~mL}$ of release medium, and placed in a constant temperature shaker at $37 \pm 0.5^{\circ} \mathrm{C}$ at $100 \mathrm{rpm}$ to maintain sink conditions. Sample aliquots $(1 \mathrm{~mL})$ were withdrawn at predetermined time intervals, and an equivalent amount of fresh artificial tear solution was added to the dissolution medium. The drug concentrations were determined using the HPLC assay method.

\section{Ex vivo Permeability Studies}

Drug permeation studies were carried out using side-byside diffusion cells by modified Franz diffusion cells. After the rabbits were sacrificed, their corneas were excised immediately and placed in iced $\left(4 \pm 1^{\circ} \mathrm{C}\right)$ artificial tear solution. The excised rabbit corneas were clamped between the donor and the receptor chamber. $2 \mathrm{~mL}$ of each formulation (E-SLNs or E-Susp) and $3 \mathrm{~mL}$ of artificial tear solution were added to the donor chamber, the mixture solution was placed close to the epithelial side of the cornea, and $5 \mathrm{~mL}$ of artificial tear solution was added to the receptor chamber. The cell top was completely sealed with parafilm to prevent evaporation. The cells were maintained at $37 \pm 0.5^{\circ} \mathrm{C}$ with magnetic stirring. The samples were collected at 15,30,60, 90, 120, 160, and $180 \mathrm{~min}$, and replaced by an equal volume of preheated fresh medium immediately at the same temperature. The samples were analyzed using HPLC as described above. The analysis method had been previously validated. Sink conditions were maintained in the receptor compartment during ex vivo permeation studies.

The cumulative amount of drug permeated per unit area $\left(\mu \mathrm{g} / \mathrm{cm}^{2}\right)$ was plotted against time $(\mathrm{min})$. The permeation enhancement was assessed with regard to permeation parameters, and the ECZ flux at $180 \mathrm{~min}\left(\mathrm{~J}_{\mathrm{ss}} ; \mu \mathrm{g} / \mathrm{cm}^{2} / \mathrm{s}\right)$ and permeability coefficient $\left(\mathrm{P}_{\mathrm{app}} ; \mathrm{cm} / \mathrm{s}\right)$ were calculated using Equations 5 and $6:{ }^{19}$

$$
\begin{gathered}
\mathrm{J}_{\mathrm{ss}}=(\Delta \mathrm{Q} / \Delta \mathrm{t}) /(\mathrm{A} \times 60) \\
\mathrm{P}_{\mathrm{app}}=\mathrm{J}_{\mathrm{ss}} / \mathrm{C}_{0}
\end{gathered}
$$

where $\mathrm{Q}$ is the total amount permeated at time t, $\Delta \mathrm{Q} / \Delta \mathrm{t}$ is the steady-state flux into the receiving solution $(\mu \mathrm{g} / \mathrm{min})$ and is equal to the gradient of the linear portion of the graph, 60 is the minutes to seconds conversion rate, A is the area of exposed cornea $\left(0.694 \mathrm{~cm}^{2}\right)$, and $\mathrm{C}_{0}$ is the initial drug concentration added to the donor chamber.

After the last sampling, the corneas were collected and the corneal hydration rate was calculated as following equation: ${ }^{20}$

$$
\text { Hydration rate }=\left(1-\mathrm{W}_{\mathrm{a}} / \mathrm{W}_{\mathrm{b}}\right) \times 100 \%
$$

where $\mathrm{W}_{\mathrm{b}}$ was the wet cornea weight and $\mathrm{W}_{\mathrm{a}}$ was the corresponding dry cornea weight after a desiccation of 8 h at $80^{\circ} \mathrm{C}$.

\section{Assessment of Antifungal Activity}

The in vitro antifungal activity of the E-SLNs and E-Susp solution was tested against thirty Fusarium clinical isolates using the broth microdilution method by following the Clinical and Laboratory Standards Institute M38-A2 document. Drug-free SLNs and pure medium were used as controls. The $50 \%$ and $90 \%$ minimum inhibitory concentrations (MICs) of the strains tested $\left(\mathrm{MIC}_{50}\right.$ and $\mathrm{MIC}_{90}$ ) were measured using SPSS Statistics (version 13.0).

The inhibition zone test was performed with one of the 30 isolates by the agar diffusion method. ${ }^{21}$ Four wells (6 $\mathrm{mm}$ in diameter) were cut into agar with a cornea ring drill, and $50 \mu \mathrm{L}$ of E-SLNs, E-Susp, blank SLNs and the positive control solution (Natamycin Ophthalmic Suspension, Natacyn ${ }^{\circledR}$ ) were placed into each well. Plates were stabilized at room temperature for $2 \mathrm{~h}$ to allow radial diffusion of the formulae and then incubated at $37^{\circ} \mathrm{C}$ for $48 \mathrm{~h}$.

\section{In vivo Ocular Irritation Study}

The ocular irritation effects of the E-SLNs eye drops were assessed according to the Draize eye test with slight modifications. ${ }^{22}$ This test was carried out in a group of three male New Zealand white rabbits, free of irritation, defects or damage. A volume of $30 \mu \mathrm{L}$ of the E-SLNs filtered through a $0.22 \mu \mathrm{m}$ PVDF membrane (Millex ${ }^{\circledR}$-GP, USA) was instilled into the conjunctival sac in the right eye every 30 $\min$ for $3 \mathrm{~h}$. The left eye was treated with physiological saline as a control. The corneal lesions and opacity (score 0-4), conjunctival chemosis, redness, discharge (score 0-3) and iris alterations (score 0-2) were microscopically observed 1, 2, 4, 24, 48 and $72 \mathrm{~h}$ after the last instillation. Ocular irritation scores for each rabbit were calculated by adding together the irritation scores for the cornea, the conjunctiva and the iris. The eye irritation score was obtained by dividing the total scores for all rabbits by the number of rabbits. A positive reaction was defined when the average numerical score 
equaled or exceeded specific numerical cutoffs, such as corneal opacity score $\geq 1$, iris score $\geq 1$ or conjunctival score $\geq 2$.

\section{In vivo Ocular Pharmacokinetic Studies in Rabbits}

Forty-two male New Zealand white rabbits (weighing 2.0 2.5 kg) free of eye diseases were chosen and divided into two groups at randomly. Each rabbit eye received 50 $\mu \mathrm{L}$ of E-SLNs filtered through a $0.22 \mu \mathrm{m}$ PVDF membrane (Millex ${ }^{\circledR}$-GP, USA) instilled in the conjunctival sac. At time intervals of 5, 15, 30,60, 120, $300 \mathrm{~min}$, tear film samples were collected by placing a sterile filter paper disc $7 \mathrm{~mm}$ in diameter under the lower eyelid of the rabbit's eye for only $1 \mathrm{~min}$ at the predetermined time. After rinsing the eye surface with saline, the rabbits were euthanized by an overdose of $4 \%$ pentobarbital sodium via ear vein injection. Then, corneal samples were harvested with surgical scissors and forceps. All corneas were rinsed with saline, blotted with filter paper and weighed. All samples were stored at $-70^{\circ} \mathrm{C}$ until they were used for extraction.

The filter paper discs containing tear liquid were thawed at room temperature and cut into two pieces with scissors. Next, $200 \mu \mathrm{L}$ of methanol was added. The disc pieces were soaked for $24 \mathrm{~h}$ and centrifuged for $10 \mathrm{~min}$ at $8000 \mathrm{rpm}$. The supernatant was transferred to sample vials for HPLC analysis. The corneas were discarded following the method reported by Li et al. ${ }^{10}$

To assess the ocular bioavailability of E-SLNs and E-Susp, the pharmacokinetic parameters were estimated using DAS2.1.1 software (Shanghai BioGuider Medicinal Technology Co., Ltd., Shanghai, China). Four main pharmacokinetic parameters were presented as the detected value: the maximum concentration $\left(\mathrm{C}_{\max }\right)$, the time $\left(\mathrm{T}_{\max }\right)$ for $\mathrm{C}_{\max }$ to occur, the elimination half-life time $\left(t_{1 / 2}\right)$ and the area under the concentration-time curve up to $180 \mathrm{~min}\left(\mathrm{AUC}_{0-180 \mathrm{~min}}\right)$.

\section{Statistical Analysis}

All the results are expressed as the mean \pm standard deviation. The groups were compared by applying twoway analysis of variance (ANOVA) using SPSS (V19), where differences were significant at $p$-values $<0.05$.

\section{Results and Discussion Physicochemical Characterization of E-SLNs}

Particle size measurements were required to confirm the production of particles in the nanometer range. As shown in Table 1, the mean diameter of the E-SLNs was $19.05 \pm$ $0.28 \mathrm{~nm}$, notably in the nanosized range, while E-Susp was about $2 \mu \mathrm{m}$. Particles smaller than $30 \mathrm{~nm}$ are considered to have the ability to penetrate across the corneal barrier. ${ }^{23,24}$ Thus, E-SLNs theoretically could penetrate the cornea, increasing the amount of drug at the site of action. PDI is used to estimate the average dispersion homogeneity, and higher PDI values correspond to broader size distribution in the dispersed sample. ${ }^{25}$ Table 1 demonstrated that the PDI of E-SLNs was only 0.21 , indicating a narrow particle size distribution, which was consistent with the particle size distribution analysis (Figure 1B). The morphology of the E-SLNs was determined by TEM (Figure 1A) and appeared to be uniform and spherical in shape with a smooth surface, signifying that the encapsulated drug vesicles were in the nanometer range with no signs of aggregation, indicating the physical stability of the E-SLNs. These results were confirmed by the subsequent stability study, as there were no changes in morphology, size or drug content within 6 months of storage at $4^{\circ} \mathrm{C}$. The size distribution, PDI and morphology of the E-SLNs indicated that the E-SLNs was a uniform and stable system.

During the preparation of the SLNs, Tween 80 was used as a surfactant. The content of Tween 80 was $20 \mathrm{mg} / \mathrm{mL}$, greater than its critical micelle concentration (CMC), which is $0.014 \mathrm{mg} / \mathrm{mL}$ at $25^{\circ} \mathrm{C}$. When the concentration of surfactant was slightly higher than its $\mathrm{CMC}$, micelles formed as spheres, which may encapsulate the drug. However, few researchers pay attention to the percentage of drug incorporated into SLNs or micelles. During preparation, E-SLNs, E-micelles and free drug could all exist in the solution. The free drug could be separated from the colloid system by ultrafiltration or dialysis. However, the drug incorporated in micelles could not be separated by these two methods. To isolate the drug in the micelles, the colloid systems were destroyed by dilution to lower the content of Tween 80 to

Table I Various Characterizations of the E-SLNs

\begin{tabular}{|l|c|c|c|c|c|}
\hline Batch & Mean Diameter (nm) & Polydispersity Index & Zeta Potential & \%EE & \% SLN \\
\hline E-SLNs & $19.05 \pm 0.28$ & $0.21 \pm 0.01$ & $-2.20 \pm 0.10$ & $94.18 \pm 1.86$ & $99.35 \pm 0.16$ \\
\hline
\end{tabular}

Note: All values are expressed as the means $\pm S D(n=3)$.

Abbreviations: \% EE, percentage entrapment efficiency; \% SLN, proportion of ECZ incorporated into SLNs. 

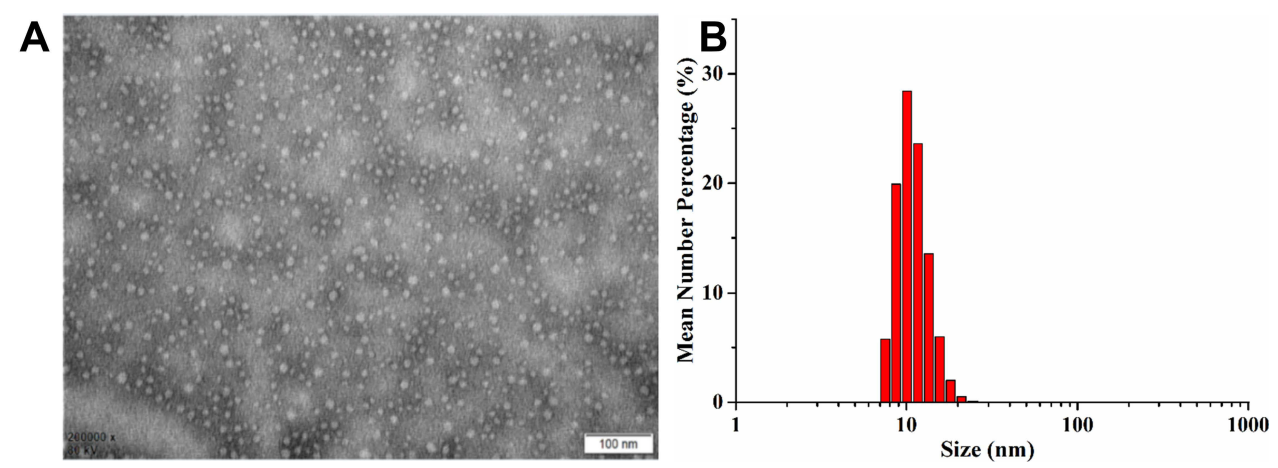

Figure I (A) Transmission electron microphotograph of E-SLNs. Bar = $100 \mathrm{~nm}$. (B) Particle size distribution of E-SLNs.

below its CMC. Then, the drug in the micelle was released, separated by ultrafiltration and quantified by HPLC. As shown in Table 1, the EE of the E-SLNs was $94.18 \pm$ $1.86 \%$, and the ratio of ECZ encapsulated in E-SLNs was $99.35 \pm 0.16 \%$, indicating that the E-SLNs were successfully prepared and almost all the ECZ was incorporated into the E-SLNs, consistent with the following DSC results.

\section{In vitro Drug Release}

The in vitro drug release profiles are important to understand and predict the in vivo performance of the formula. Figure 2 depicted an obvious slow and controlled release of ECZ from the E-SLNs, with approximately $98 \%$ of the drug released after $96 \mathrm{~h}$. This controlled release may be attributed to the biodegradation and surface erosion of the lipid matrix. ${ }^{6}$ The burst effect is a common problem associated with SLNs release because drug attached to the surface of SLNs might be expelled during storage due to the use of surfactant. ${ }^{26}$ However, no burst effect was found in the release of E-SLNs, largely due to lower surfactant concentrations during preparation. ${ }^{27}$

Kinetic analysis of the ECZ release data from E-SLNs was performed depending on the calculated correlation coefficient $\left(r^{2}\right)$ for each kinetic model (Table 2). The $r^{2}$ of the E-SLNs fit best to the Higuchi model. The in vitro release study may be evidence that the drug is homogeneously dispersed in the solid-lipid core of $\mathrm{SLNs}^{28}$ and release through the lipid matrix and/or biodegradation and surface erosion of the lipid matrix, ${ }^{29}$ as confirmed by the next DSC study.

\section{Differential Scanning Calorimetry (DSC)}

DSC was used to investigate the melting and recrystallization behavior of the substance. The DSC thermograms of the samples are presented in Figure 3. The DSC

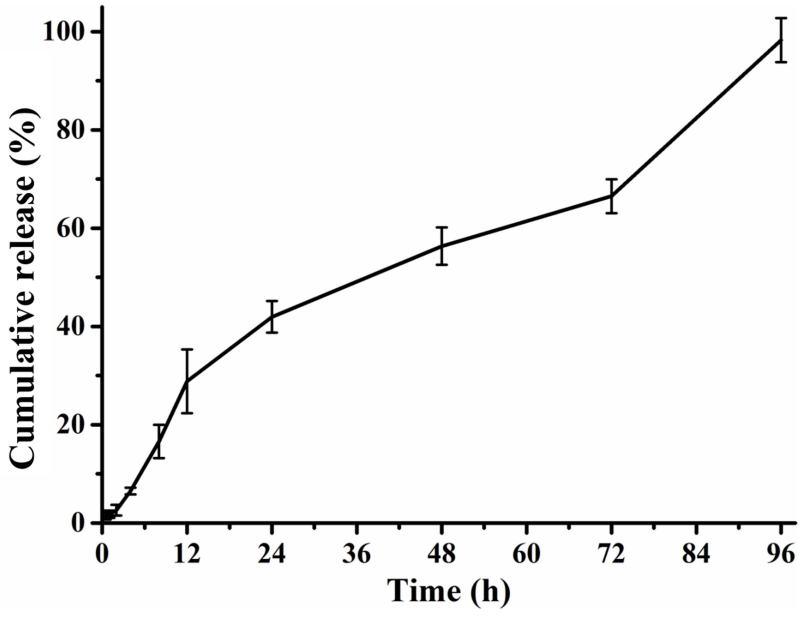

Figure 2 In vitro release profiles of E-SLNs ( $\mathrm{pH} 7.4$ artificial tear solution was used as the dialysis medium, $\mathrm{n}=3$, mean $\pm S D$ ).

thermograms of ECZ and tripalmitin showed sharp melting endotherms at $89.48^{\circ} \mathrm{C}$ and $58.76^{\circ} \mathrm{C}$, respectively. The physical mixture of ECZ and tripalmitin showed characteristic peaks of both ECZ and tripalmitin. The DSC curve of the lyophilized E-SLNs showed a small endotherm at $57.66^{\circ} \mathrm{C}$, which corresponds to the melting point of tripalmitin. However, the ECZ melting endotherm was disappeared in the thermogram of E-SLNs, probably due to the dissolution of the crystals into the molten lipid. ${ }^{30}$ This suggests that ECZ was present in an amorphous state and entirely entrapped

Table 2 Mathematical Models of Regression for the in vitro Release Profiles of E-SLNs

\begin{tabular}{|l|c|c|}
\hline Kinetic Model & Equation & $\boldsymbol{r}^{2}$ \\
\hline Zero order & $y=0.93 x+5.85$ & 0.9744 \\
First order & $y=\mid 14.33-\mathrm{e}^{4.74-0.015 x}$ & 0.9830 \\
Higuchi & $y=8.57\left(x^{\wedge}(I / 2)\right)+1$ & 0.9900 \\
\hline
\end{tabular}




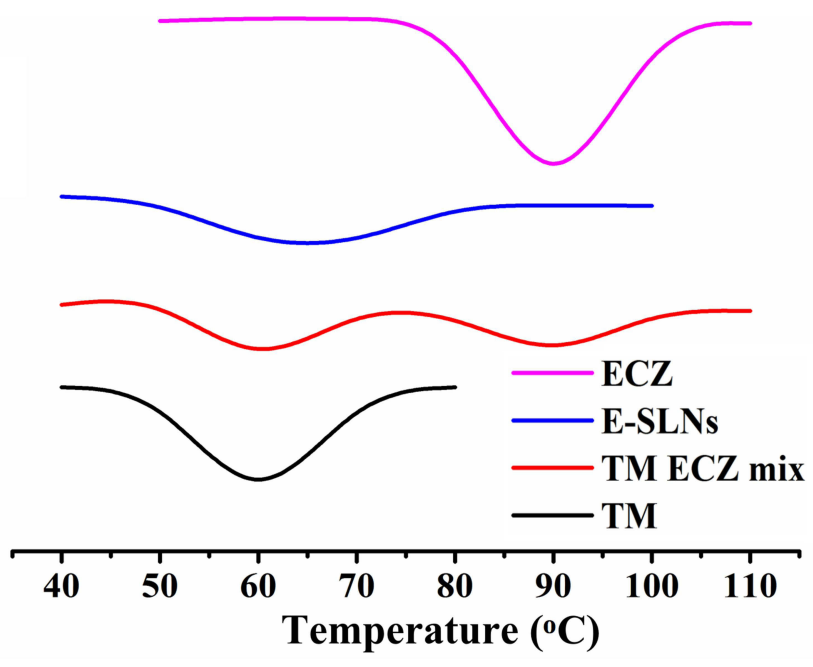

Figure 3 DSC thermograms of tripalmitin (TM), econazole (ECZ), E-SLNs and a physical mixture of ECZ and TM.

within the lipid matrix, as rapid quenching of the microemulsion and the presence of surfactant do not allow the drug to crystallize in an amorphous state, and the drug was entirely entrapped in the lipid matrix. ${ }^{31}$

\section{Ex vivo Drug Permeation Studies}

Ex vivo corneal permeation studies were carried out to evaluate the permeation of E-SLNs and E-Susp in the rabbit cornea. At the end of the corneal permeation study, the corneal hydration level was evaluated.

As shown in Table 3, E-SLNs increased the apparent corneal permeability coefficient $(\mathrm{P} a p p)(7.13 \pm 0.05)$ by approximately 2.64 -fold compared to E Susp $(1.96 \pm 0.23)$ because the particle size of E-SLNs was smaller than the porosity of the tight junctions exiting the corneal epithelium. ${ }^{32}$ E-SLNs could enter and then penetrate the cornea, facilitated by the concentration gradient between the donor and receptor chambers, which is consistent with a previous report. ${ }^{33}$

Corneal hydration is an important parameter to assess damage to corneal tissue. The hydration level of a healthy cornea should be between 75 and $80 \%{ }^{34}$ Irreversible damage to corneal tissues may occur once the corneal hydration level reaches $>83 \% .{ }^{35}$ In this experiment, the hydration rate of the corneas in the E-SLN group was $76.25 \pm 2.29 \%$, while that in the E-Susp group was $89.13 \pm 1.15 \%$, indicating that the cornea in E-Susp suffered from irreversible harm. So, E-Susp could be harmful to the cornea while E-SLNs were relatively safe to the cornea. These results were consistent with the results of the subsequent irritation experiment.

\section{Antifungal Susceptibility Testing}

Antifungal assays were performed by using agar diffusion and microdilution methods ${ }^{36}$ to determine whether the SLNs enhanced the antifungal activity of ECZ. As shown in Table 4 and Figure 4, the diameter of the inhibition zone of E-Susp against the Fusarium clinical isolates was $18 \pm$ $1 \mathrm{~mm}$, while that for E-SLNs was $25 \pm 1 \mathrm{~mm}$. The $\mathrm{MIC}_{50}$ and $\mathrm{MIC}_{90}$ of E-Susp against the Fusarium clinical isolates were 2.43 and 1.75 folds of E-SLNs. Antifungal testing showed that the E-SLNs possessed enhanced antifungal activity compared with E-Susp. ${ }^{37}$

\section{Irritation Studies}

In general, ocular irritation is a key factor affecting ophthalmic drug development and clinical use. SLNs have been used to decrease drug irritation as a result of their small size. ${ }^{6}$ In this work, the irritancy of E-SLNs was evaluated in New Zealand white rabbits. As shown in Figure 5, no signs of ocular irritancy were detected compared with the control group. The results of the Draize test showed that the use of SLNs as a vehicle could eliminate the irritancy of ECZ suspensions to the eye. ${ }^{38}$

\section{Ocular Pharmacokinetics of ECZ in Rabbit Eyes}

The ECZ concentrations in the tear liquid versus time after single topical administrations of E-SLNs and E-Susp are shown in Figure 6A. Both E-SLNs and E-Susp were immediately absorbed into the tear liquid to reach maximum concentration $\left(\mathrm{C}_{\max }\right)$ at $5 \mathrm{~min}$, and the ECZ concentration decreased markedly in the following hours, which was caused by the loss of the drug in the conjunctival sac and the tear wash. The ECZ levels in the tear film of E-Susp eyes were significantly higher than those in

Table 3 Corneal Permeation Parameters of the ECZ from E-SLNs and E-Susp and the Hydration Rates

\begin{tabular}{|l|l|l|l|}
\hline Group & Papp*10 $\left.\mathbf{~}^{\mathbf{5}} \mathrm{cm} \mathrm{s}^{-1}\right)$ & $J^{-1} * 10^{3}\left(\mu \mathrm{g} \mathrm{s}^{-1} \mathrm{~cm}\right)$ & Hydration Rate (\%) \\
\hline E-SLNs & $7.13 \pm 0.05$ & $4.28 \pm 0.03$ & $76.25 \pm 2.29$ \\
E-Susp & $1.96 \pm 0.23$ & $1.29 \pm 0.15$ & $89.13 \pm 1.15$ \\
\hline
\end{tabular}

Note: All values are expressed as means \pm SD $(n=3)$. 
Table 4 Comparative Study of the Antifungal Activity of E-SLNs and E-Susp Against A.Fusarium

\begin{tabular}{|l|l|l|l|}
\hline Formulation & Mean of Diameter of Zone of Inhibition $(\mathbf{m m})$ & MIC $_{\mathbf{5 0}}(\boldsymbol{\mu g} / \mathbf{m L})$ & MIC \\
\hline E-SLNs & $25 \pm 1$ & 0.37 & 0.89 \\
E-Susp & $18 \pm 1$ & 0.90 & 1.56 \\
\hline
\end{tabular}

Note: All values are expressed as the means \pm SD $(n=3)$.

Abbreviations: $\mathrm{MIC}_{50}$, minimal inhibitory concentration for $50 \%$ of the strains tested; $\mathrm{MIC}_{90}$, minimal inhibitory concentration for $90 \%$ of the strains tested.

E-SLNs eyes at both $5 \mathrm{~min}$ and $15 \mathrm{~min}$, and the trend was reversed after $30 \mathrm{~min}$ because of the sustained-release properties of E-SLNs reside in conjunctival sac and cornea. Thus, the ECZ concentration decline curve for

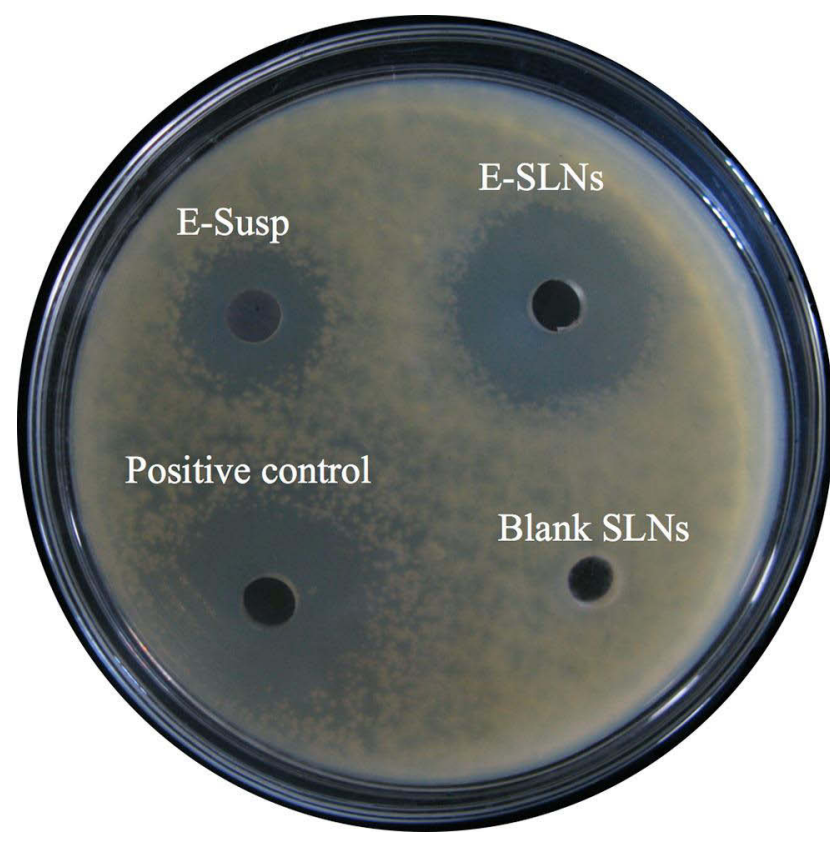

Figure 4 Microbiological studies against A.Fusarium by the agar diffusion method for $48 \mathrm{~h}$.
E-SLNs was gentle compared to that of E-Susp due to the controlled release of ECZ from the SLNs, also displayed in vitro release test. As Table 5 shows, E-SLNs produced a higher mean residual time (MRT) (1.58-fold) than E-Susp benefit from that the SLNs ensured adherence to the surrounding membranes in the conjunctival sac and prolonged the retention time on the ocular surface.

As shown in Figure 6B, E-SLNs produced significantly higher drug concentrations in the cornea than E-Susp at all determined time points $(p<0.01)$. Both E-SLNs and E-Susp achieved $\mathrm{C}_{\max }$ immediately after the application of the formula; however, E-SLNs produced higher $\mathrm{C}_{\max }$ (3.7-fold), $\mathrm{AUC}_{(0-180 \mathrm{~min})}$ (2.9-fold) and MRT (1.3-fold) values than E-Susp as shown in Table 5, indicating that the use of SLNs as a drug delivery system could greatly improve the ocular bioavailability of ECZ compared to traditional preparations.

The ECZ concentrations in the cornea after a single administration of E-SLNs were all above the $\mathrm{MIC}_{90}$ during the determined time period, signifying that another administration is not necessary for at least three hours. Nevertheless, after $90 \mathrm{~min}$, the corneal drug concentration of $\mathrm{ECZ}$ from E-Susp was less than the $\mathrm{MIC}_{90}$, indicating the interval between two doses should be less than 90 minutes. Therefore, E-SLNs showcased advantage of reducing the frequency of administration.
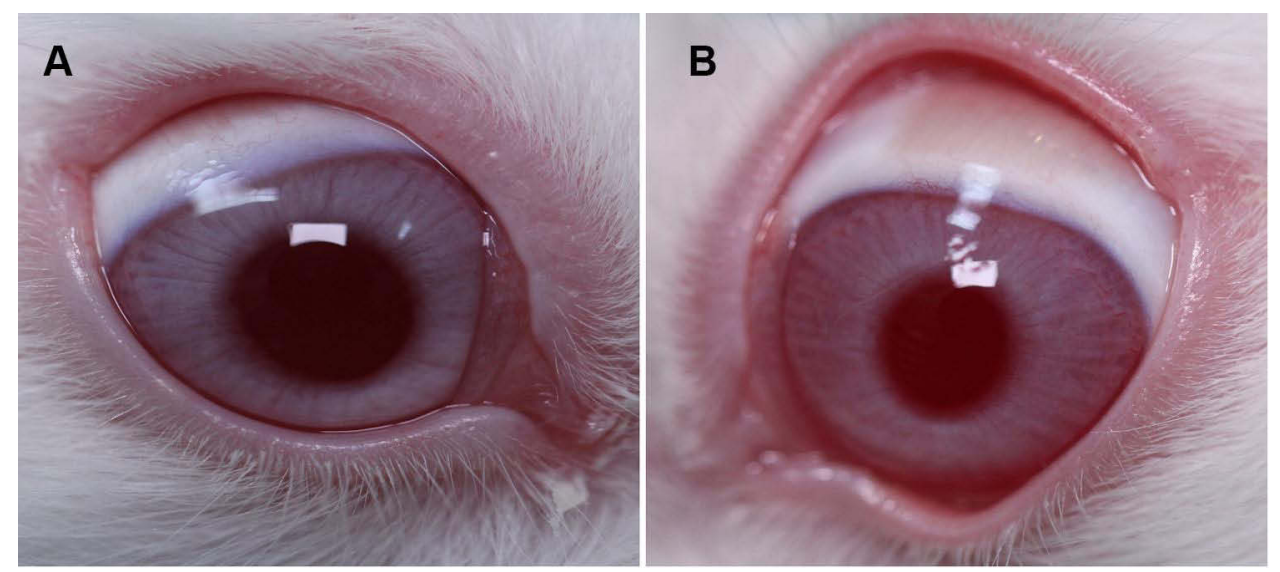

Figure 5 Representative images of the in vivo rabbit ocular irritation test. (A) E-SLNs, (B) Saline. 

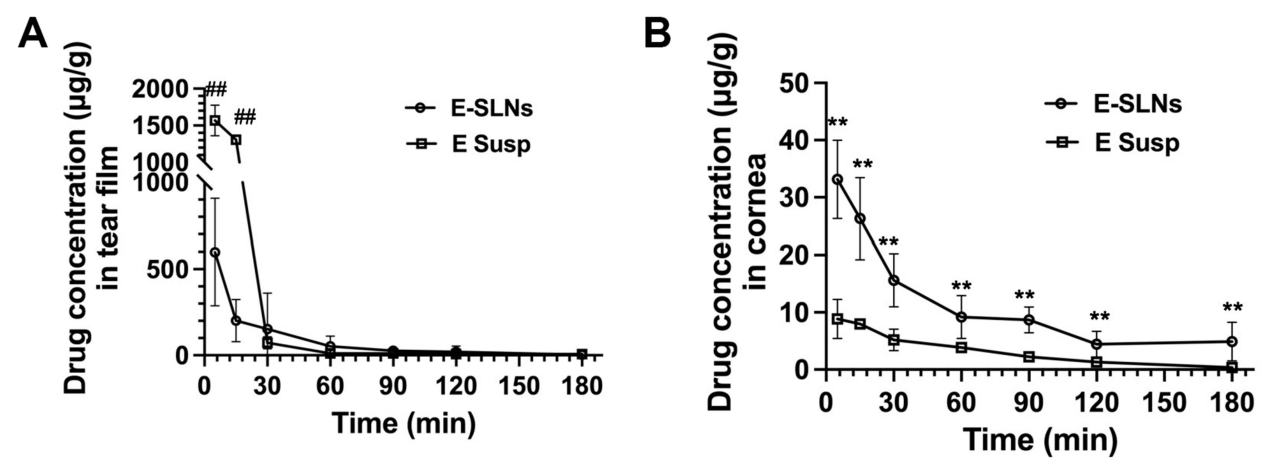

Figure 6 Pharmacokinetics studies in rabbit eyes after a single administration of either formulation. The ECZ concentration-time profiles in the (A) Tear film and (B) Corneas of rabbits. The drugs were E-SLNs and E-Susp at a single dose of $50 \mu \mathrm{L}$. Values are given as the mean \pm SD $(n=6)$. **Significantly different ( $p<0.0 \mathrm{I})$ from the E-Susp

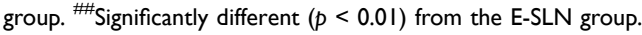

ECZ might penetrate the cornea as a free drug or in the form of E-SLNs. Tween 80 exert inhibitory effects on certain drug efflux transporters (P-glycoproteins). ${ }^{39}$ Nevertheless, after $90 \mathrm{~min}$, the corneal drug concentration of ECZ from E-Susp was less than the $\mathrm{MIC}_{90}$ due to its large size $(2 \mu \mathrm{m})$. The conjunctiva is a conduit for drug clearance into systemic circulation, and the conjunctival route is important for large molecules that are not able to penetrate through the corneal barrier. ${ }^{40}$ Considering this theory and the ECZ concentration in the tear film, E-Susp was largely removed by the conjunctiva.

The effective drug concentration of antimycotic drugs in lesion locations of FK is essential. However, due to precorneal loss factors, including tear dynamics, turnover, transient residence time in the cul-de-sac, nonproductive absorption and relative impermeability of the corneal epithelial membrane, the bioavailability of topical ocular drugs is very poor. ${ }^{41}$ To achieve therapeutic efficacy, increasing the frequency of administration during clinical treatment, which is inconvenient to patients and causes serious adverse reactions in the ocular surface. ${ }^{42}$ SLNs are therefore a potential drug carrier system with the advantages of high bioavailability and a lower frequency of drug administration.

\section{Conclusion}

In this study, E-SLNs for ocular use with an appropriate mean particle size, PDI, EE\%, SLN\% and sustained release were successfully prepared by the microemulsion method. E-SLNs exhibited good bioavailability and prolonged residence time in the cornea and enhanced antifungal activity compared with E-Susp with no irritation. This study could be a promising approach for ocular delivery of antifungal drugs for treatment of ocular fungal keratitis.

Table 5 Ocular Pharmacokinetic Parameters of E-SLNs and E-Susp in the Tear Liquid and Cornea After the Administration of Different Formulations

\begin{tabular}{|l|l|l|l|}
\hline \multicolumn{2}{|l|}{ Pharmacokinetic Parameters } & \multicolumn{2}{c|}{ Formulations } \\
\cline { 3 - 4 } & & E-SLNs & E-Susp \\
\hline \multirow{2}{*}{ Tear film } & $T_{\max }(\mathrm{min})$ & 5 & 5 \\
& $C_{\max }(\mathrm{mg} / \mathrm{L})$ & $597.64 \pm 309.77$ & $1570.54 \pm 208.2 I^{\#}$ \\
& $M R T_{(0-t)}\left(\mathrm{mg} / \mathrm{L}^{*} \min \right)$ & 50.25 & 31.74 \\
& $A U C_{(0-180 \min )}$ & $30,921.78$ \\
\hline \multirow{2}{*}{ Cornea } & $T_{\max }(\min )$ & $13,957.45$ & 5 \\
& $C_{\max }(\mathrm{mg} / \mathrm{L})$ & 5 & $8.85 \pm 3.42$ \\
& $M R T_{(0-t)}(\mathrm{mg} / \mathrm{L*} \min )$ & $33.15 \pm 6.82^{* *}$ & 35.29 \\
& $A U C_{(0-180 \min )}$ & 43.99 & 536.1 \\
\hline
\end{tabular}

Notes: All values are expressed as the means $\pm S D(n=3) ; C_{\max }$ the maximum concentration of drug in eye tissue, $T_{\max }$ time required to reach the maximum eye tissue concentration, AUC the area under eye tissue concentration-time curve from 0 to $180 \mathrm{~min}$, MRT mean residue time. $* *$ Considered significant compared to E-Susp ( $p<$ 0.0I). ${ }^{\#}$ Considered significant compared to E-SLNs $(p<0.0 \mathrm{I})$. 


\section{Abbreviations}

ECZ, Econazole; E-SLNs, solid-lipid nanoparticle-based ECZ eye drops; E-Susp, ECZ Suspension; FK, Fungal keratitis; SLNs, Solid lipid nanoparticles; EE, entrapment efficiency; CMC, critical micelle concentration; HPLC, highperformance liquid chromatography; PDI, polydispersity index; TEM, Transmission electron microscopy; DSC, Differential scanning calorimetry; $\mathrm{MIC}_{50}$, minimal inhibitory concentration for $50 \%$ of the strains tested; $\mathrm{MIC}_{90}$, minimal inhibitory concentration for $90 \%$ of the strains tested; $\mathrm{C}_{\max }$, the maximum concentration; $\mathrm{T}_{\max }$, the time for $\mathrm{C}_{\max }$ to occur; $\mathrm{t}_{1 / 2}$, the elimination half-life time; $\mathrm{AUC}_{0-180 \mathrm{~min}}$, the area under the concentration-time curve up to $180 \mathrm{~min}$.

\section{Acknowledgments}

The authors are grateful to Dr. Junxia Zhang (Henan Provincial Institute of Food and Drug Control) for her assistance with the animal housing.

\section{Funding}

This research was supported by the National Natural Science Foundation of China [U1704283 and 52173143] and the Fundamental and Frontier Technological Program of Henan Province, China [142300410057].

\section{Disclosure}

The authors have declared that there are no conflicts of interest with this article.

\section{References}

1. Brown L, Leck AK, Gichangi M, et al. The global incidence and diagnosis of fungal keratitis. Lancet Infect Dis. 2021;21(3):E49-E57. doi:10.1016/S1473-3099(20)30448-5

2. Shahram M, Ahmad M, Kazem A, et al. Fungal keratitis: an overview of clinical and laboratory aspects. Mycoses. 2018;61(5):916-930. doi: $10.1111 /$ myc. 12822

3. Sha X, Shi Q, Liu L, et al. Update on the management of fungal keratitis. Int Ophthalmol. 2021;41(9):3249-3256.

4. Lin Y, Zhang J, Han X, et al. A retrospective study of the spectrum of fungal keratitis in southeastern China. Ann Palliat Med. 2021;10 (9):9480-9487.

5. Chang HY, Chodosh J. Diagnostic and therapeutic considerations in fungal keratitis. Int Ophthalmol Clin. 2011;51(4):33-42. doi:10.1097/ IIO.0b013e31822d64dc

6. Khames A, Khaleel MA, El-Badawy MF, et al. Natamycin solid lipid nanoparticles-sustained ocular delivery system of higher corneal penetration against deep fungal keratitis: preparation and optimization. Int J Nanomed. 2019;14:2515-2531. doi:10.2147/IJN.S190502

7. Velpandian T, Nirmal J, Sharma HP, et al. Novel water soluble sterile natamycin formulation (Natasol) for fungal keratitis. Eur J Pharm Sci. 2021;163:105857. doi:10.1016/j.ejps.2021.105857

8. Shi L, Li Z, Liang Z, et al. A dual-functional chitosan derivative platform for fungal keratitis. Carbohydr Polym. 2021;275:118762. doi:10.1016/j.carbpol.2021.118762
9. Díaz-Tomé V, Luaces-Rodríguez A, Silva-Rodríguez J, et al. Ophthalmic econazole hydrogels for the treatment of fungal keratitis. J Pharm Sci. 2018;107(5):1342-1351. doi:10.1016/j. xphs.2017.12.028

10. Li J, Li Z, Liang Z, et al. Fabrication of a drug delivery system that enhances antifungal drug corneal penetration. Drug Deliv. 2018;25 (1):938-949. doi:10.1080/10717544.2018.1461278

11. Bachu RD, Chowdhury P, Al-Saedi ZHF, et al. Ocular drug delivery barriers-role of nanocarriers in the treatment of anterior segment ocular diseases. Pharmaceutics. 2018;10(1):28-58. doi:10.3390/ pharmaceutics 10010028

12. Jain D, Carvalho E, Banerjee R. Biodegradable hybrid polymeric membranes for ocular drug delivery. Acta Biomater. 2010;6 (4):1370-1379. doi:10.1016/j.actbio.2009.11.001

13. Singh M, Guzman-Aranguez A, Hussain A, et al. Solid lipid nanoparticles for ocular delivery of isoniazid: evaluation, proof of concept and in vivo safety \& kinetics. Nanomedicine. 2019;14(4):465-491. doi:10.2217/nnm-2018-0278

14. Shah RM, Eldridge D, Palombo EA, et al. Optimisation and stability assessment of solid lipid nanoparticles using particle size and zeta potential. J Phys Ther Sci. 2014;25(1):59-75.

15. Sánchez-López E, Espina M, Doktorovova S, et al. Lipid nanoparticles (SLN, NLC): overcoming the anatomical and physiological barriers of the eye-Part I-barriers and determining factors in ocular delivery. Eur J Pharm Biopharm. 2017;110:70-75. doi:10.1016/j. ejpb.2016.10.009

16. Sánchez-López E, Espina M, Doktorovova S, et al. Lipid nanoparticles (SLN, NLC): overcoming the anatomical and physiological barriers of the eye-Part II-ocular drug-loaded lipid nanoparticles. Eur J Pharm Biopharm. 2017;110:58-69. doi:10.1016/j. ejpb.2016.10.013

17. Chetoni P, Burgalassi S, Monti D, et al. Solid lipid nanoparticles as promising tool for intraocular tobramycin delivery: pharmacokinetic studies on rabbits. Eur J Pharm Biopharm. 2016;109:214-223. doi:10.1016/j.ejpb.2016.10.006

18. Liu S, Han $\mathrm{X}$, Liu $\mathrm{H}$, et al. Incorporation of ion exchange functionalized-montmorillonite into solid lipid nanoparticles with low irritation enhances drug bioavailability for glaucoma treatment. Drug Deliv. 2020;27(1):652-661. doi:10.1080/10717544.2020.1756984

19. Younes NF, Abdel-Halim SA, Elassasy AI. Solutol HS15 based binary mixed micelles with penetration enhancers for augmented corneal delivery of sertaconazole nitrate: optimization, in vitro, ex vivo and in vivo characterization. Drug Deliv. 2018;25(1):1706-1717. doi:10.1080/10717544.2018.1497107

20. Vega E, Gamisans F, García ML, et al. PLGA nanospheres for the ocular delivery of flurbiprofen: drug release and interactions. J Pharm Sci. 2008;97(12):5306-5317. doi:10.1002/jps.21383

21. Malhotra S, Khare A, Grover K, et al. Design and evaluation of voriconazole eye drops for the treatment of fungal keratitis. J Pharm. 2014;2014:490595.

22. Barroso J, Pfannenbecker U, Adriaens E, et al. Cosmetics Europe compilation of historical serious eye damage/eye irritation in vivo data analysed by drivers of classification to support the selection of chemicals for development and evaluation of alternative methods/ strategies: the Draize eye test Reference Database (DRD). Arch Toxicol. 2017;91(2):521-547.

23. Baba K, Tanaka Y, Kubota A, et al. A method for enhancing the ocular penetration of eye drops using nanoparticles of hydrolyzable dye. J Control Release. 2011;153(3):278-287. doi:10.1016/j. jconrel.2011.04.019

24. Li J, Li Z, Zhou T, et al. Positively charged micelles based on a triblock copolymer demonstrate enhanced corneal penetration. Int J Nanomed. 2015;10:6027-6037. doi:10.2147/IJN.S90347

25. Li Z, Liu R, Guo Z, et al. Celastrol-based nanomedicine promotes corneal allograft survival. J Nanobiotechnol. 2021;19(1):341. doi:10.1186/s12951-021-01079-w 
26. Almeida H, Amaral MH, Lobao P, et al. Applications of polymeric and lipid nanoparticles in ophthalmic pharmaceutical formulations: present and future considerations. J Pharm Pharm Sci. 2014;17 (3):278-293. doi:10.18433/J3DP43

27. Garcês A, Amaral MH, Lobo J, et al. Formulations based on solid lipid nanoparticles (SLN) and nanostructured lipid carriers (NLC) for cutaneous use: a review. Eur J Pharm Sci. 2017;112:159-167. doi:10.1016/j.ejps.2017.11.023

28. Bhatt R, Singh D, Prakash A, et al. Development, characterization and nasal delivery of rosmarinic acid-loaded solid lipid nanoparticles for the effective management of huntington's disease. Drug Deliv. 2015;22(7):931-939. doi:10.3109/10717544.2014.880860

29. Abd El-Gawad AEH, Soliman OA, El-Dahan MS, et al. Improvement of the ocular bioavailability of econazole nitrate upon complexation with cyclodextrins. AAPS PharmSciTech. 2017;18(5):1795-1809. doi:10.1208/s12249-016-0609-9

30. Mohanty B, Majumdar DK, Mishra SK, et al. Development and characterization of itraconazole-loaded solid lipid nanoparticles for ocular delivery. Pharm Dev Technol. 2015;20(4):458-464. doi:10.3109/10837450.2014.882935

31. Khare A, Singh I, Pawar P, et al. Design and evaluation of voriconazole loaded solid lipid nanoparticles for ophthalmic application. J Drug Deliv. 2016;2016:6590361. doi:10.1155/2016/6590361

32. Rajapaksha A, Fink M, Todd BA. Size-dependent diffusion of dextrans in excised porcine corneal stroma. Mol Cell Biomech. 2015;12 (3):215-230.

33. Bisht R, Mandal A, Jaiswal JK, et al. Nanocarrier mediated retinal drug delivery: overcoming ocular barriers to treat posterior eye diseases. Wiley Interdiscip Rev Nanomed Nanobiotechnol. 2018;10:2.

34. Kumar R, Sinha VR. Solid lipid nanoparticle: an efficient carrier for improved ocular permeation of voriconazole. Drug Dev Ind Pharm. 2016;42(12):1956-1967. doi:10.1080/03639045.2016.1185437
35. Li X, Nie SF, Kong J, et al. A controlled-release ocular delivery system for ibuprofen based on nanostructured lipid carriers. Int $J \quad$ Pharm. 2008;363(1-2):177-182. doi:10.1016/j. ijpharm.2008.07.017

36. Fernandes F, Salgado H. Antimicrobial activity of spondias dulcis Parkinson extract leaves using microdilution and agar diffusion: a Comparative Study. EC Microbiol. 2019;15(4):259-262.

37. Yousry C, Zikry PM, Salem HM, et al. Integrated nanovesicular/ self-nanoemulsifying system (INV/SNES) for enhanced dual ocular drug delivery: statistical optimization, in vitro and in vivo evaluation. Drug Deliv Transl Res. 2020;3:801-814. doi:10.1007/s13346-02000716-5

38. Nagai N, Ito Y, Okamoto N, et al. A nanoparticle formulation reduces the corneal toxicity of indomethacin eye drops and enhances its corneal permeability. Toxicology. 2014;319:53-62.

39. Prabhakar K, Afzal SM, Surender G, et al. Tween 80 containing lipid nanoemulsions for delivery of indinavir to brain. Acta Pharm Sin B. 2013;3(5):345-353. doi:10.1016/j.apsb.2013.08.001

40. Guo Z, Shi L, Feng H, et al. Reduction-sensitive nanomicelles: delivery celastrol for retinoblastoma cells effective apoptosis. Chin Chem Lett. 2021;32(3):1046-1050. doi:10.1016/j.cclet.2020.03.066

41. Li Z, Guo Z, Chu D, et al. Effectively suppressed angiogenesis-mediated retinoblastoma growth using celastrol nanomicelles. Drug Deliv. 2020;27(1):358-366. doi:10.1080/ 10717544.2020 .1730522

42. Li Z, Zhang X, Guo Z, et al. Nature-derived bionanomaterials for sustained release of 5-fluorouracil to inhibit subconjunctival fibrosis. Mater Today Adv. 2021;11:100150. doi:10.1016/j.mtadv.2021.100150
International Journal of Nanomedicine

\section{Publish your work in this journal}

The International Journal of Nanomedicine is an international, peerreviewed journal focusing on the application of nanotechnology in diagnostics, therapeutics, and drug delivery systems throughout the biomedical field. This journal is indexed on PubMed Central, MedLine, CAS, SciSearch ${ }^{\circledR}$, Current Contents ${ }^{\circledR} /$ Clinical Medicine,

\section{Dovepress}

Journal Citation Reports/Science Edition, EMBase, Scopus and the Elsevier Bibliographic databases. The manuscript management system is completely online and includes a very quick and fair peer-review system, which is all easy to use. Visit http://www.dovepress.com/ testimonials.php to read real quotes from published authors. 